\title{
TIME-OPTIMAL PATHS FOR LATERAL NAVIGATION OF AN AUTONOMOUS UNDERACTUATED AIRSHIP
}

\author{
Salim Hima and Yasmina Bestaoui \\ Laboratoire Systèmes Complexes, CNRS-FRE 2492, Université d'Evry Val d'Essonne \\ 38, Rue du Pelvoux, 91020 Evry, France \\ \{hima, bestaoui\}@iup.univ-evry.fr
}

\begin{abstract}
This paper deals with a characterization of the shortest paths for lateral navigation of an autonomous underactuated airship taking into account its dynamics and actuator limitations. The initial and terminal positions are given. We would like to specify the control forces that steer the unmanned aerial vehicle to the given terminal position requiring the minimal time for lateral navigation. The application of Pontryagin's Maximal Principle, allows us to find a family of timeoptimal paths. Based on the symmetry of airship dynamics, i.e. with respect to rotation and translation, it is possible to construct global trajectories connecting two configurations by a succession of a finite number of these time-optimal paths using geometric reasoning.
\end{abstract}

\section{INTRODUCTION}

Unmanned aerial vehicles are a new focus of research, because of their important application potential. They can be divided into three different types : reduced scale fixed wing vehicles (airplanes), rotary wing aircraft (helicopter) or lighter than air vehicles (airships). Lighter than air vehicles suit a wide range of applications, ranging from advertising, aerial photography and survey work tasks. They are safe, costeffective, durable, environmentally benign and simple to operate. Airships offer the advantage of quiet hover with noise levels much lower than helicopters. Unmanned remotely-operated airships have already proved themselves as camera and TV platforms, surveillance and for specialized scientific tasks such as earth monitoring and environmental control. An actual trend is toward autonomous airships.

What makes a vehicle lighter than air is the fact that it uses a lifting gas (i.e. helium or hot air) in order to be lighter than the surrounding air. The principle of Archimedes applies in the air as well as under water. Airships are powered and have some means of controlling their direction. Non rigid airships are the most common form nowadays. They are basically large gas balloons. The most common form of a dirigible is an ellipsoid. It is a highly aerodynamically profile with good resistance to aerostatics pressures. Its shape is maintained by its internal overpressure. The only solid parts are the gondola, the set of propeller (a pair of propeller mounted at the gondola) and the tail fins. The envelope holds the helium that makes the blimp lighter than air. In addition to the lift provided by helium, airships derive aerodynamic lift from the shape of the envelope as it moves through the air.

The objective of this paper is to generate a desired flight trajectory to be followed by the airship. The trajectory generation module generates a nominal state trajectory and a nominal control input. A mission starts with take-off from the platform where the mast that holds the mooring device of the airship is mounted. Typically, flight operation modes can be defined as: take-off, cruise, landing and hover. After the user has defined the goal tasks, the path generator then determines a path for the vehicle that is a trajectory in space. In Aeronautics, plane flight control often involves lateral and longitudinal state decoupling. The problem of trajectory generation for lateral control is formulated as an optimization problem. This motion generation takes into account the constraints on velocity and the bound on the rudder angle. The minimum time problem is solved using the maximum principle of Pontryagin. Once this reference trajectory determined, the airship can follow it with an appropriate feedback.

The lighter than air platform of the 'Laboratoire des Systèmes Complexes' is the AS200 by Airspeed Airships. It is a remotely piloted airship designed for remote sensing. It is a non rigid $6 \mathrm{~m}$ long, $1.4 \mathrm{~m}$ diameter and $8.6 \mathrm{~m}^{3}$ volume airship equipped with two vectorable engines on the sides of the gondola and 4 control surfaces at the stern. The four stabilizers are externally braced on the full and rudder movement is provided by direct linkage to the servos. Envelope pressure is maintained by air fed from the propellers into the two ballonets located inside the central portion of the hull. These ballonets are self regulating and can be fed from either engine. The engines are standard model aircraft type units. 


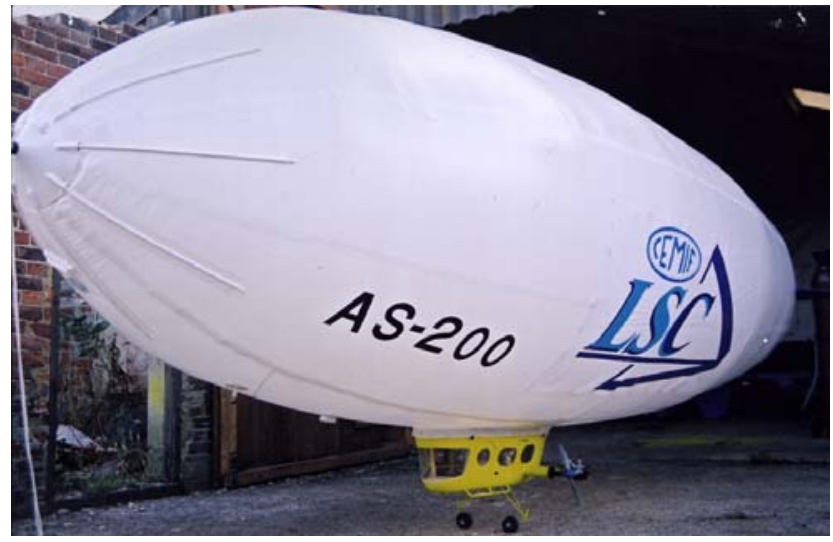

Figure1 LSC airship platform AS200

\section{AIRSHIP DYNAMIC MODELING}

\section{Kinematic modeling}

A general spatial displacement of a rigid body consists of a finite rotation about a spatial axis and a finite translation along some vector. The rotational and translational axes in general need not be related to each other. It is often easiest to describe a spatial displacement as a combination of a rotation and a translation motions, where the two axes are not related. However, the combined effect of the two partial transformations (i.e. rotation, translation about their respective axes) can be expressed as an equivalent unique screw displacement, where the rotational and translational axes in fact coincide. The concept of a screw thus represents an ideal mathematical tool to analyze spatial transformation. The finite rotation of a rigid body does not obey to the laws of vector addition (in particular commutativity) and as a result the angular velocity of the body cannot be integrated to give the attitude of the body. There are many ways to describe finite rotations. Direction cosines, Rodriguez Hamilton's (quaternions) variables, Euler parameters, Euler angles, can serve as examples. Some of these groups of variables are very close to each other in their nature. The usual minimal representation of orientation is given by a set of three Euler angles, assembled with the three position coordinates allow the description of the situation of a rigid body. A direction cosine matrix (of Euler rotations) is used to describe the orientation of the body (achieved by 3 successive rotations) with respect to some fixed frame reference.

Three reference frames are considered, figure 2 , in the derivation of the kinematics and dynamics equations of motion. These are the Earth fixed frame $R_{f}$ considered as Galilean, and two local frames attached to airship, the body fixed frame $R_{m}$ and aeronautic frame $R_{a}$. The position and orientation of the vehicle should be described relative to the inertial reference frame while the linear and angular velocities of the vehicle should be expressed in the body-fixed coordinate system. This formulation has been first used for underwater vehicles. In this paper, the origin $C$ of $R_{m}$ coincides with the center of volume of the vehicle. Its axes are the principal axes of symmetry when available. They must form a right handed orthogonal normed frame.

The $x_{a}$ axis of the aeronautic frame follows the direction of the airship relative velocity $V_{r}$ with respect to the wind. $\alpha$ is the angle of attack within the $x_{m} z_{m}$ plane, and $\beta$ the skid angle within the $x_{m} y_{m}$ plane. To describe the position and the orientation of the airship

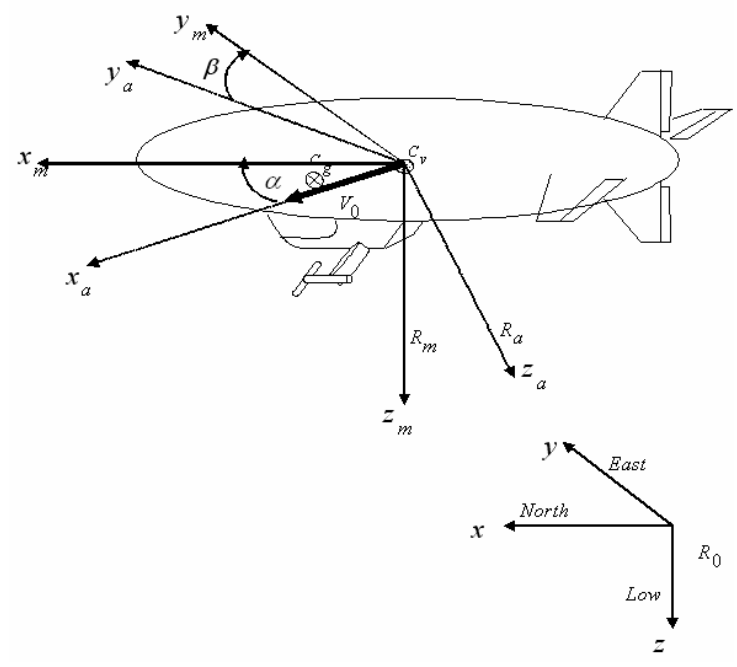

Figure2 General configuration of frames

with respect to the inertial reference frame $R_{f}$, the Eulerian parameterization is used. The three orientation angles are: the Roll $\phi$, the Pitch $\theta$ and the Yaw $\psi$. The current configuration is then deduced from three elementary rotations. The position $\eta_{1}$ and the orientation $\eta_{2}$ of the vehicle in $R_{f}$ can be respectively described by:

$$
\eta_{1}=\left(\begin{array}{lll}
x & y & z
\end{array}\right)^{T} \text { and } \eta_{2}=\left(\begin{array}{lll}
\phi & \theta & \psi
\end{array}\right)^{T}
$$

Then the orientation matrix $H_{\lambda}$ between the body fixed frame $R_{m}$ and reference $R_{f}$ is given by ${ }^{2,4}$ : 


$$
\begin{aligned}
& H_{\lambda}=\left(\begin{array}{cc}
C \psi C \theta & -S \psi C \phi+C \psi S \theta S \phi \\
S \psi C \theta & C \psi C \phi+S \psi S \theta S \phi \\
-S \theta & S \phi C \theta
\end{array}\right. \\
& \left.\begin{array}{c}
S \psi S \phi+C \psi S \theta C \phi \\
-C \psi S \phi+S \psi S \theta C \phi \\
C \phi C \theta
\end{array}\right)
\end{aligned}
$$

and the transform matrix $H_{a}$ between the body fixed frame $R_{m}$ and the aeronautic frame $R_{a}$ can be written as:

$$
H_{a}=\left(\begin{array}{ccc}
C \alpha C \beta & -C \alpha S \beta & -S \alpha \\
S \beta & C \beta & 0 \\
S \alpha C \beta & -S \alpha S \beta & C \alpha
\end{array}\right)
$$

where $C x=\cos (x)$ and $S x=\sin (x)$

$H_{a}$ and $H_{\lambda} \in S O(3)$ denotes the rotation matrix that specifies the orientation of the airship frame relative to the inertial reference frame in inertial reference frame coordinates. $S O(3)$ is the special orthogonal group of order 3 which is represented by the set of all $3 \times 3$ orthogonal rotation matrices that characteristics are ${ }^{11}$ :

$$
R R^{T}=I_{3 \times 3} \text { and } \operatorname{det}(R)=1
$$

$I_{3 \times 3}$ represents the $3 \times 3$ identity matrix.

This description is valid in the region $-\frac{\pi}{2} \leq \theta \leq \frac{\pi}{2}$. A singularity of this transformation exists for:

$$
\theta=\frac{\pi}{2} \pm k \pi, k \in \mathrm{Z}
$$

Let's now introduce $V=\left(\begin{array}{lll}u & v & w\end{array}\right)^{T}$ as the linear velocity of the origin $C$ expressed in $R_{m}$ and $\Omega=\left(\begin{array}{lll}p & q & r\end{array}\right)^{T}$ as the angular velocity expressed in that frame. The kinematics of the airship can be expressed in the following way:

$$
\dot{\eta}=\left(\begin{array}{c}
\dot{\eta_{1}} \\
\dot{\eta_{2}}
\end{array}\right)=\left(\begin{array}{cc}
R & 0_{3 \times 3} \\
0_{3 \times 3} & J\left(\eta_{2}\right)
\end{array}\right)\left(\begin{array}{l}
V \\
\Omega
\end{array}\right)
$$

where

$$
J\left(\eta_{2}\right)=\left(\begin{array}{ccc}
1 & S \phi \tan \theta & C \phi \\
0 & C \phi & -S \phi \\
0 & S \phi / C \theta & C \phi / C \theta
\end{array}\right)
$$

\section{Dynamic modeling}

In this section, analytic expressions for the forces and moments acting on the airship are derived. It is advantageous to formulate the equations of motion in a body fixed frame to take advantage of the vehicle's geometrical properties. Applying Newton's laws of motion relating the applied forces and moments to the resulting translational and rotational accelerations assembles the equations of motion for the 6 degrees of freedom. The forces and moments are referred to a system of body-fixed axes, centered at the airship center of volume. We will make in the sequel some simplifying assumptions: the earth fixed reference frame is inertial, the gravitational field is constant, the airship is supposed to be well inflated, the aeroelastic effects are ignored, the density of air is supposed to be uniform, and the influence of gust is considered as a continuous disturbance, ignoring its stochastic character. The deformations are considered to be negligible.

Global dynamics equation can be written as ${ }^{4}$ :

$$
M_{d} \stackrel{\ddot{\eta}}{=}=\sum \vec{\tau}_{\text {ext }}+\vec{\tau}_{m}(\eta)
$$

where $M_{d}$ and $\vec{\tau}_{m}(\eta)$ are respectively the inertia matrix and the dynamical (Coriolis and centrifugal) tensors which are due to the mass of airship, and $\sum \vec{\tau}_{\text {ext }}$ is the sum of the different external tensor, which involve:

- Global aerodynamic tensor due to the added mass phenomenon plus forces and moments generated by the airship body (hull, fins and gondola).

- Aerostatic tensor describing the forces and moments due to the gravity and buoyancy

- Propulsion tensor due to the vectored thrust.

\section{Lateral dynamics of the airship}

The airship model consists of 12 states, complicating the control design. In aeronautics area, a very natural simplification consists of decomposing the flight modes into: take off, cruise and landing. These tasks can be divided into two main modes: longitudinal mode and lateral one. In this paper we focus on lateral mode in constant altitude. The vectored thrusters and elevators are associated to the longitudinal navigation controller to hold the altitude and relative velocity navigation constant. The rudders allow the airship to navigate in the horizontal plan.

The mathematical model of the trimmed lateral dynamics in the local frame is given by ${ }^{6}$ :

$$
\begin{aligned}
& m_{y} v-\left(m a_{z}+\dot{Y}_{p}\right) \dot{p}+\left(m a_{x}-\dot{Y_{r}}\right) \dot{r} \\
& =m_{z} W_{e} p-m_{x} U_{e} r+Y_{e}+\dot{Y}_{v} v+\dot{Y}_{p} p \\
& +\dot{Y}_{r} r+\dot{Y}_{\delta} \delta_{r}+\left(F_{G}-F_{B}\right) \phi \cos \left(\theta_{e}\right)
\end{aligned}
$$




$$
\begin{aligned}
& J_{z} \dot{r}-J_{x z} \dot{p}+\left(m a_{x}-N_{v}\right) \dot{v}=m a_{x} W_{e} p \\
& -m a_{x} U_{e} r+N_{e}+N_{v} v+N_{p} p+\dot{N}_{r} r \\
& +\dot{N}_{\delta} \delta_{r}+a_{x} F_{G} \phi \cos \left(\theta_{e}\right) \\
& J_{x} \dot{p}-J_{x z} \dot{r}-\left(m a_{z}+\dot{L}_{v}\right) \dot{v}=-m a_{z} W_{e} p \\
& +m a_{z} U_{e} r+L_{e}+\dot{L}_{v} v+\dot{L}_{p} p+\dot{L}_{r} r \\
& -a_{x} F_{G} \phi \cos \left(\theta_{e}\right)
\end{aligned}
$$

these equations correspond to the Lateral, Yaw and roll dynamics. $U_{e}$ and $\mathrm{v}$ are the axial and lateral velocity components in local frame.

$$
\begin{aligned}
U_{e} & =V_{r} \cos (\beta) \\
v & =V_{r} \sin (\beta)
\end{aligned}
$$

where $\dot{Y}_{x}, Y_{x}, \dot{N}_{x}, N_{x}, \dot{L}_{x}$ and $L_{x}$ are the aerodynamic coefficients. $m_{i}$ is the apparent mass in the $\mathrm{i}^{\text {th }}$ direction. $J_{i}$ are inertia matrix elements. $m$ is the airship mass. $\theta_{e}$ is the equilibrium pitch angle.

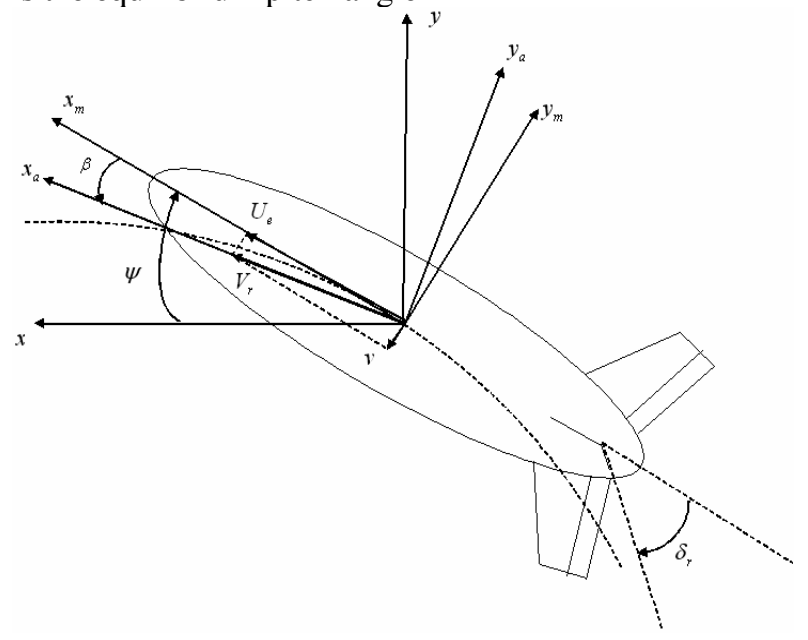

Figure3 Lateral configuration of airship (top view)

$a_{i}$ are the coordinates of the center of mass in the local frame $R_{m}{ }^{6}$. $\beta$ is the skid angle between the relative velocity $V_{r}$ and $x_{m}$ axis into $x_{m} y_{m}$ plane ${ }^{5}$. In the absence of wind, this angle appears when the airship follows a path with a non zero curvature. For a fixed rudder deflection, i.e. corresponding to the circle path, this angle takes a constant value when an equilibrium between aerodynamic moment, caused by the airship body (hull, the vertical fins and control surfaces) motion with respect to the surrounding air and the centrifugal one is established. This angle takes on small values.
In general, the airship moves with a low speed. The equilibrium between the centrifugal moment around $x_{m}$ axis caused by the rudder deflection and gravitational moment is the cause of an insignificantly small Roll angle and rate which can be omitted. Taking these considerations into account, the model can be simplified as:

$$
\begin{aligned}
m_{y} \dot{v}+\left(m a_{x}-\dot{Y_{r}}\right) \dot{r}= & -m_{x} U_{e} r+\dot{Y}_{v} v+\dot{Y}_{r} r \\
& +\dot{Y}_{\delta} \delta_{r} \\
J_{z} \dot{r}+\left(m a_{x}-\dot{N}_{v}\right) \dot{v} & =-m a_{x} U_{e} r++\dot{N}_{v} v \\
& +N_{r} r+\dot{N}_{\delta} \delta_{r}
\end{aligned}
$$

and the kinematic equations are given by:

$$
\begin{aligned}
& \psi=r \\
& \dot{x}=V_{r} \cos (\psi+\beta) \\
& \dot{y}=V_{r} \sin (\psi+\beta)
\end{aligned}
$$

Recapitulating, the model of the lateral dynamics of the airship can be written as:

$$
\begin{aligned}
& \dot{\beta}=a_{11} \beta+a_{12} r+b_{1} \delta_{r} \\
& \dot{r}=a_{21} \beta+a_{22} r+b_{2} \delta_{r} \\
& \dot{\psi}=r \\
& \dot{x}=V_{r} \cos (\psi+\beta) \\
& \dot{y}=V_{r} \sin (\psi+\beta)
\end{aligned}
$$

where:

$$
\begin{aligned}
& a_{11}=\frac{J_{z} \dot{Y}_{v}+\left(-m a_{x}+\dot{Y}_{r}\right) \dot{N}_{v}}{m_{y} J_{z}-\left(m a_{x}-\dot{Y_{r}}\right)\left(m a_{x}-\dot{N_{v}}\right)} \\
& a_{12}=\frac{J_{z}\left(\dot{Y}_{r}-m_{x} U_{e}\right)+\left(-m a_{x}+\dot{Y}_{r}\right)\left(\dot{N}_{r}-m a_{x} U_{e}\right)}{m_{y} J_{z}-\left(m a_{x}-\dot{Y_{r}}\right)\left(m a_{x}-N_{v}\right)}
\end{aligned}
$$




$$
a_{21=} \frac{\left(m a_{x}-\dot{N}_{v}\right) \dot{Y_{v}}+m_{y} \dot{N}_{v}}{m_{y} J_{z}-\left(m a_{x}-\dot{Y_{r}}\right)\left(m a_{x}-\dot{N_{v}}\right)} \quad \begin{aligned}
& \dot{X}=f(X)+g(X) u \\
& X\left(t_{0}\right)=X_{0} \text { and } X\left(t_{f}\right)=X_{f} \\
& u_{\min } \leq u \leq u_{\max }
\end{aligned}
$$$$
a_{22}=\frac{\left(m a_{x}-\dot{N_{v}}\right)\left(\dot{Y}_{r}-m_{x} U_{e}\right)+m_{y}\left(\dot{N}_{r}-m a_{x} U_{e}\right)}{m_{y} J_{z}-\left(m a_{x}-\dot{Y_{r}}\right)\left(m a_{x}-\dot{N_{v}}\right)}
$$$$
b_{1}=\frac{J_{z} \dot{Y}_{\delta}-\left(\dot{Y_{r}}-m a_{x}\right) \dot{N}_{\delta}}{m_{y} J_{z}-\left(m a_{x}-\dot{Y_{r}}\right)\left(m a_{x}-\dot{N_{v}}\right)}
$$$$
b_{2}=\frac{\left(m a_{x}-\dot{N}_{v}\right) \dot{Y}_{\delta}+m_{y} \dot{N}_{\delta}}{m_{y} J_{z}-\left(m a_{x}-\dot{Y_{r}}\right)\left(m a_{x}-\dot{N_{v}}\right)}
$$

The lateral dynamics of the airship have an affine structure. In the compact form the dynamics can be given by:

$$
X=f(X)+g \delta_{r}
$$

where :

$$
f(X)=\left(\begin{array}{c}
a_{11} \beta+a_{12} r \\
a_{21} \beta+a_{22} r \\
r \\
V_{r} \cos (\beta+\psi) \\
V_{r} \sin (\beta+\psi)
\end{array}\right) \text { and } g=\left(\begin{array}{c}
b_{1} \\
b 2 \\
0 \\
0 \\
0
\end{array}\right)
$$

Some difficulties arise with this model: the first one is the underactuation of this system, i.e. 5 states steered by a single input control. The second one is the nonholonomic character: non integrable relationship between velocities:

$$
x \sin (\beta+\psi)+y \cos (\beta+\psi)=0
$$

this kind of constraints are called Pfaffian Constraints ${ }^{8}$.

\section{Time Optimal Extremals}

In this paragraph, let's introduce reference timeoptimal paths for the system under study, taking into account the system dynamics and actuator capabilities. Hence, this problem can be formulated as follows:

$$
\min \int_{t_{0}}^{t_{f}} d t=t_{f}-t_{0}
$$

Subject to
The problem is to find the admissible control $u$ that minimize the time for which the system reaches the final state $X_{f}$ from the initial one $X_{0}$. Without loss of generality, and by a simple normalization and shifting (if the two bounds of the control domain are not symmetric), we can constrain the control to belong to a unit interval, i.e. $-1 \leq u \leq 1$.

To solve this problem, we apply the Pontryagin's Maximum Principle $(P M P)$ to obtain necessary conditions for a reference trajectory of a system to be time-optimal. The $P M P$ states that: if $X(t)$ is timeoptimal trajectory defined on $[0, T]$, and $u(t)$ is the corresponding time-optimal reference control, then there exists an absolutely continuous vector function called the adjoint vector, $\lambda:[0, T] \rightarrow \mathfrak{R}^{5}$, such that the following conditions are satisfied ${ }^{1,7,9}$ :

1. $\lambda(t) \neq 0$ for all $t \in[0, T]$

2.

$$
\begin{aligned}
H(X(t), \lambda(t), u(t))= & \min _{v} H(X(t), \lambda(t), v(t)) \\
= & -\lambda_{0} \leq 0
\end{aligned}
$$

with $\lambda_{0}>0$ for every fixed $X(t), \lambda(t)$ and $t$, such that $H(X(t), \lambda(t), u(t))=\lambda_{0}+\left\langle\lambda^{T}(t), X(t)\right\rangle$, where $\langle\cdot, \cdot\rangle$ is the vector space inner product.

3. the adjoint vector $\lambda(t)$ satisfies the following equation: $\dot{\lambda}=-\frac{\partial H}{\partial X}$

A triple $(X, \lambda, u)$ verifying the necessary conditions is called an extremal. First, consider the Hamiltonian $H$, functional for the optimal control problem where $\lambda$ are multipliers that adjoin the constraints.

The Hamiltonian function of the system is given by:

$$
\begin{aligned}
& H\left(X(t), \lambda(t), \delta_{r}(t)\right)=\lambda_{0}+\lambda^{T} f(X)+\lambda^{T} g(X) \delta_{r} \\
& =\lambda_{0}+\lambda_{1}\left(a_{11} \beta+a_{12} r+b_{1} \delta_{r}\right) \\
& +\lambda_{2}\left(a_{21} \beta+a_{22} r+b_{2} \delta_{r}\right)+\lambda_{3} r+\lambda_{4} V_{r} \cos (\psi+\beta) \\
& +\lambda_{5} V_{r} \sin (\psi+\beta)
\end{aligned}
$$

and the co-state dynamics are given by: 


$$
\begin{aligned}
\dot{\lambda}_{1} & =-\lambda_{1} a_{11}-\lambda_{2} a_{21}+\lambda_{4} V_{r} \sin (\psi+\beta) \\
& -\lambda_{5} V_{r} \cos (\psi+\beta) \\
\dot{\lambda}_{2} & =-\lambda_{1} a_{12}-\lambda_{2} a_{22}-\lambda_{3} \\
\dot{\lambda}_{3} & =\lambda_{4} V_{r} \sin (\psi+\beta)-\lambda_{5} V_{r} \cos (\psi+\beta) \\
\dot{\lambda}_{4} & =0 \\
\dot{\lambda}_{5} & =0
\end{aligned}
$$

Therefore, as $\lambda_{4}$ and $\lambda_{5}$ are constant on $[0, T]$ there exists $\mu \geq 0$ and $\gamma \in[0,2 \pi]$ such that, $\forall t \in[0, T]$ then :

$$
\begin{aligned}
& \dot{\lambda}_{1}=-\lambda_{1} a_{11}-\lambda_{2} a_{21}+V_{r} \mu \sin (\psi+\beta-\gamma) \\
& \dot{\lambda}_{2}=-\lambda_{1} a_{12}-\lambda_{2} a_{22}-\lambda_{3} \\
& \dot{\lambda}_{3}=V_{r} \mu \sin (\psi+\beta-\gamma) \\
& \lambda_{4}=\mu \cos (\gamma) \\
& \lambda_{5}=\mu \sin (\gamma)
\end{aligned}
$$

and the Hamiltonian becomes:

$$
\begin{aligned}
& H\left(X, \lambda, \delta_{r}\right)=\lambda_{0}+\lambda_{1}\left(a_{11} \beta+a_{12} r\right) \\
& +\lambda_{2}\left(a_{21} \beta+a_{22} r\right)+\lambda_{3} r \\
& +\mu V_{r} \cos (\psi+\beta-\gamma)+\left(\lambda_{1} b_{1}+\lambda_{2} b_{2}\right) \delta_{r}
\end{aligned}
$$

The minimization of the Hamiltonian with respect to the control $\delta_{r}$ is obtained by minimizing $\lambda^{T} g(X) \delta_{r}$. The control belongs to a unit control domain, then this minimization can be achieved by taking for $\delta_{r}$ the opposite sign of $\lambda^{T} g(X)$, then:

$$
\begin{cases}\delta_{r}=1 & \text { if } \lambda^{\mathrm{T}} g<0 \\ \delta_{r}=-1 & \text { if } \lambda^{\mathrm{T}} g>0\end{cases}
$$

The function $\phi(t)=\lambda^{T} g(X)$, defined along an extremal $\left(X, \lambda, \delta_{r}\right)$ is called the switching function associated to that system. Clearly, the zeroes of this function are important for the study of optimal synthesis. If there exists a nonempty interval such that $\phi(\cdot)$ is identically zero, the extremal is singular on that interval. Assume now the extremal to be bang, i.e. takes its values in $\{-1,1\}$ for almost a time $t_{s}$ such that $\delta_{r}$ is not almost everywhere constant on any interval of the form ]$t_{s}-\varepsilon, t_{s}+\varepsilon\left[, \varepsilon>0 . t_{s}\right.$ is called a switching time for $\delta_{r}$ and corresponding state is called switching state.

The trajectories corresponding to the controls $\delta_{r}= \pm 1$, figure 4 are circles. The fastest way to turn is turning with the smallest radius, means that making the rudders deflection in one of its limits. i.e. to turn left or right. figure5 and 6 shows, $\beta$ and $r$ curves when the airship pass from a straight line to circle. They take constant values when the circle permanent path is attained.

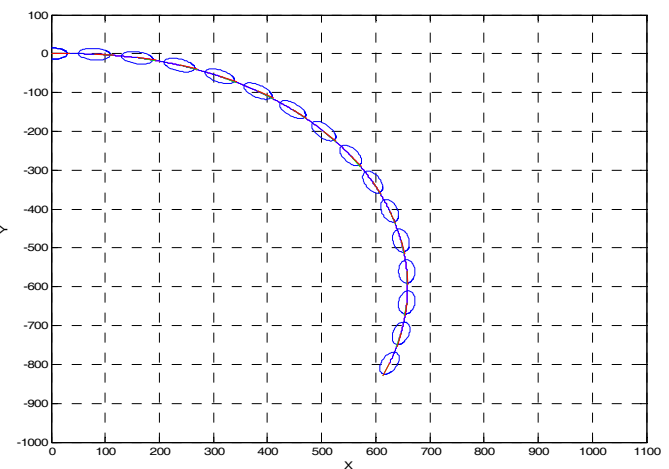

Figure 4 Circle path for $\delta_{r}=1$

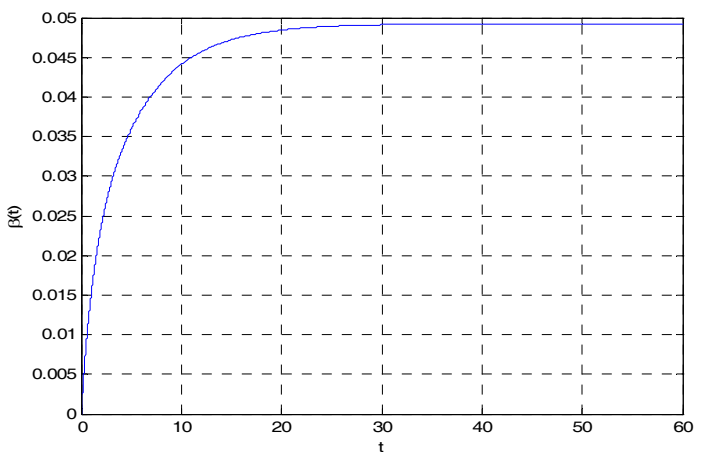

Figure 5 skid angle $\beta$ response for $\delta_{r}=1$

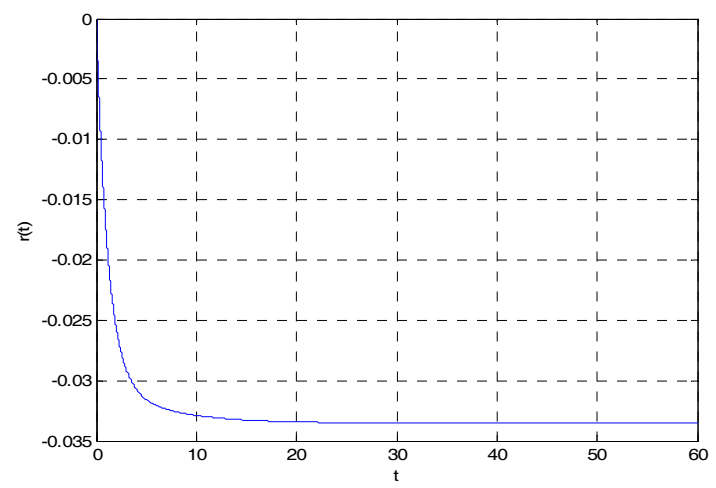

Figure6 Yaw rate response for $\delta_{r}=1$

\section{Singular extremals}

As mentioned above, the singular controls are characterized by the fact that $\phi(t)$ is identically zero in a nonempty interval. However, the PMP loses its discrimination nature, i.e. every controls in $U$ satisfy the necessary conditions. In this case, we need some additional conditions. The nullity of $\phi(t)$ in a nonempty 
interval implies that all its time derivatives are null in that interval, i.e.

$$
\dot{\phi}(t)=\ddot{\phi}(t)=\cdots=\phi^{2 m}(t)=0
$$

the process of derivation is stopped when the control appear in the expression of these derivation. For an affine system,

$$
\phi^{2 k}(t)=a(X, \lambda)+b(X, \lambda) \delta_{r}=0
$$

$k$ is called the order of singular control. Hence, the singular control can be expressed as:

$$
\delta_{r}=-\frac{a(X, \lambda)}{b(X, \lambda)}
$$

\section{Proposition1:}

The singular controls of our system are of the first order and are never abnormal.

Proof:

Let's derive the switching function $\phi(t)$ :

$$
\begin{aligned}
\phi(t) & =\lambda^{T} g=b_{1} \lambda_{1}+b_{2} \lambda_{2} \\
\dot{\phi}(t) & =\dot{\lambda}^{T} g \\
& =-\lambda^{T}\left(f_{x}+g_{x} \delta_{r}\right) g \\
& =-\lambda^{T} f_{x} g \\
& =\lambda^{T} q \\
& =b_{1} \dot{\lambda}_{1}+b_{2} \dot{\lambda}_{2}
\end{aligned}
$$

and

$$
\begin{aligned}
\ddot{\phi}(t) & =\lambda^{T} q_{x} g \delta_{r}+\lambda^{T}\left(q_{x} f-f_{X} q\right) \\
& =b_{1} \ddot{\lambda} 1+b_{2} \ddot{\lambda} 2
\end{aligned}
$$

where $V_{x}$ denotes the jacobian matrix of the vector $V$. Note that $g_{x}=0$. Nullifying these equations, we find:

$$
\begin{aligned}
& \lambda_{2}=-\frac{b_{1}}{b_{2}} \lambda_{1} \\
& \dot{\lambda}_{2}=-\frac{b_{1}}{b_{2}} \dot{\lambda}_{1}
\end{aligned}
$$

replacing in the first and the second equations of the system of equations (25), we find:

$$
\dot{\lambda}_{1}=-\lambda_{1}\left(a_{11}-\frac{b_{1}}{b_{2}} a_{21}\right)+\dot{\lambda}_{3}
$$

$$
\dot{\lambda}_{1}=-\lambda_{1}\left(a_{22}-\frac{b_{2}}{b_{1}} a_{12}\right)+\frac{b_{2}}{b_{1}} \lambda_{3}
$$

\section{proposition2:}

The system of differential equations (35), (36), has as solution $\lambda_{1}=\lambda_{3}=0$.

\section{Proof:}

From the condition $\lambda_{1}=\lambda_{3}=0$ we find,

$$
\sin (\psi+\beta-\gamma)=0
$$

implying

$$
\psi+\beta-\gamma=k \pi
$$

with $k \in Z$

this result represents the necessary condition for the existence of the singular control.

From the equation (38), we can state that, the control coefficient

$$
\lambda^{T} q_{x} g=-b_{1}^{2} V_{r} \cos (\psi+\beta-\gamma)
$$

is never vanishing.

So the Hamiltonian becomes:

$$
\begin{aligned}
H\left(X, \lambda, \delta_{r}\right) & =\lambda_{0}+\mu V_{r} \cos (\psi+\beta-\gamma) \\
& =0
\end{aligned}
$$

from this,

$$
\mu=-\frac{\lambda_{0}}{V_{r} \cos (\psi+\beta-\gamma)}= \pm \frac{\lambda_{0}}{V_{r}}
$$

we can conclude from equation (41) that: $\mu$ is never equal to zero, because the zero value of $\mu$ implies a null adjoint vector $\lambda$, this contradicts $P M P$ statements.

To prove the minimality of the singular controls, we must test the generalized convexity condition often called, strengthened Legendre -Clebsh condition given by ${ }^{3,10}$ :

$$
(-1)^{k} \frac{\partial}{\partial u}\left(\frac{d^{2 k} \phi}{d t^{2 k}}\right) \geq 0
$$

$k$ is the order of singular control. For our system:

$$
(-1) \frac{\partial}{\partial \delta_{r}}\left(\frac{d^{2} \phi}{d t^{2}}\right)=-b_{1}^{2} \mu V_{r} \cos (\psi+\beta-\gamma)
$$

from the equation (41):

$$
-b_{1}^{2} \mu V_{r} \cos (\psi+\beta-\gamma)=b_{1}^{2} \lambda_{0} \geq 0
$$

which is true, i.e. $\lambda_{0}$ is positive by definition. 
Hence, the singular control, and from equation (32) can be given by:

$$
\begin{aligned}
\delta_{r} & =-\frac{\lambda^{T}\left(q_{x} f-f_{x} q\right)}{\lambda^{T} q_{x} g} \\
& =\left(a_{11}+\frac{b_{2}}{b_{1}}\left(1+a_{12}\right)\right) \tan (\psi+\beta-\gamma) \\
& -\frac{\left(a_{11} \beta+\left(1+a_{12}\right) r\right)}{b_{1}}
\end{aligned}
$$

from the existence condition of the singular control, i.e. $\psi+\beta-\gamma=k \pi$, the control can be reduced to:

$$
\delta_{r}=-\frac{\left(a_{11} \beta+\left(1+a_{12}\right) r\right)}{b_{1}}
$$

such that $\left|\delta_{r}\right|<1$

\section{Discussion of the singular control}

Once the singular control is determined, we illustrate the geometric shape of the reference trajectory of the airship under this singular control. From the singular control necessary condition, $\psi+\beta-\gamma$ is constant, and $\gamma$ is constant too, then $\psi+\beta$ is constant. This implies that the angle between the relative velocity $V_{r}$ and the $x$ reference axis is constant. Thus the singular trajectory is a straight line. We can find the same result by applying the control defined below in the dynamics of $\beta$ :

$$
\begin{aligned}
& \beta+r=0 \\
& \dot{\beta}+\dot{\psi}=0
\end{aligned}
$$

implying a non variation of $\beta+\psi$ angle, the reference trajectory is thus a line. Transition from a curved path to the line requires the application of the singular control which grows monotonously, figure7. This explosion of control is due to the non acceptance of the discontinuities in path curvature by the airship dynamics. For this reason we want to smooth this curvature by optimizing the transition time between a non zero curvature trajectory and a straight line. When we try to connect two aligned configuration the optimal path is obviously a straight line, i.e. $\beta=r=0$, which correspond to $\delta_{r}^{*}=0$, this control is embedded in (46) .

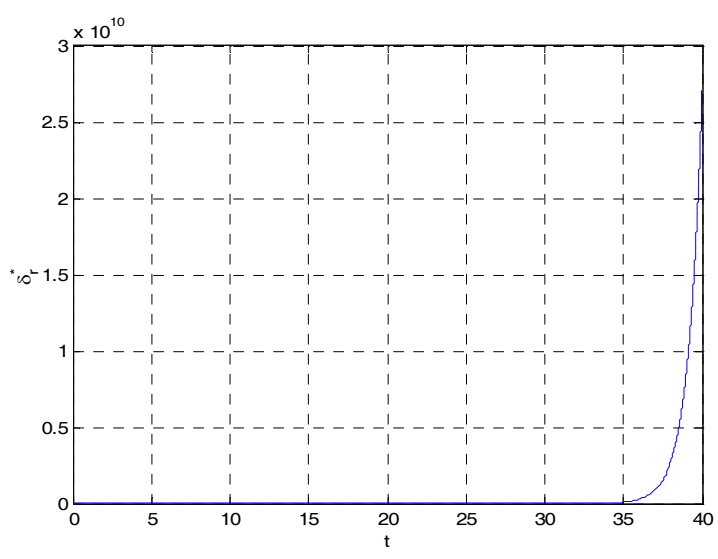

Figure7 singular control $\delta_{r}^{*}$

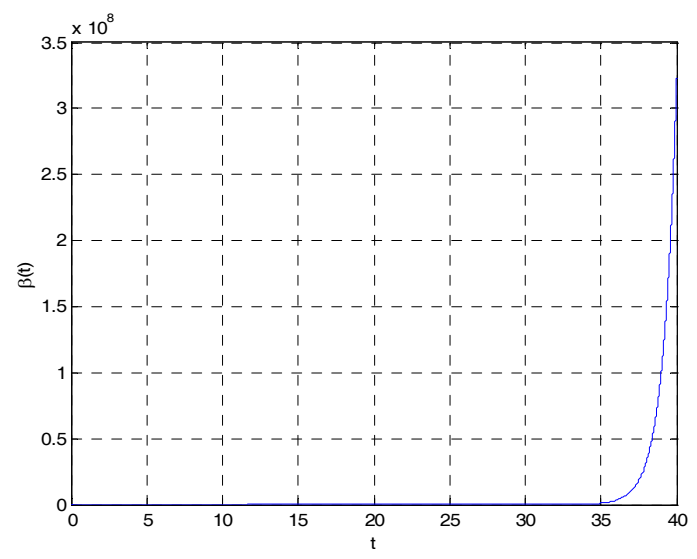

Figure 8 skid angle response to $\delta_{r}^{*}$

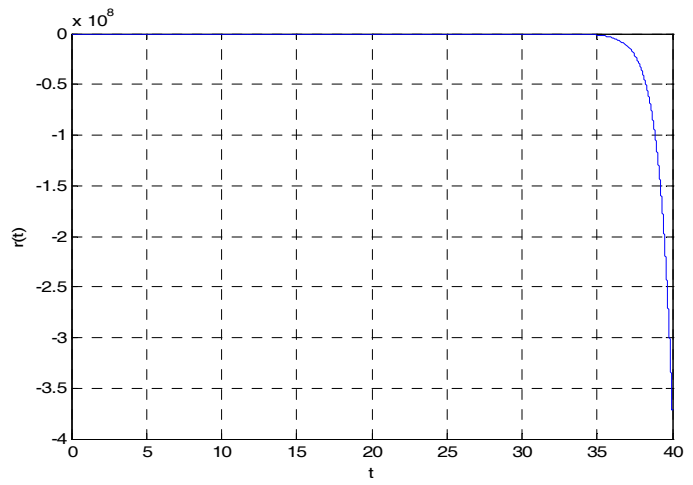

Figure 9 Yaw rate response to $\delta_{r}^{*}$ 


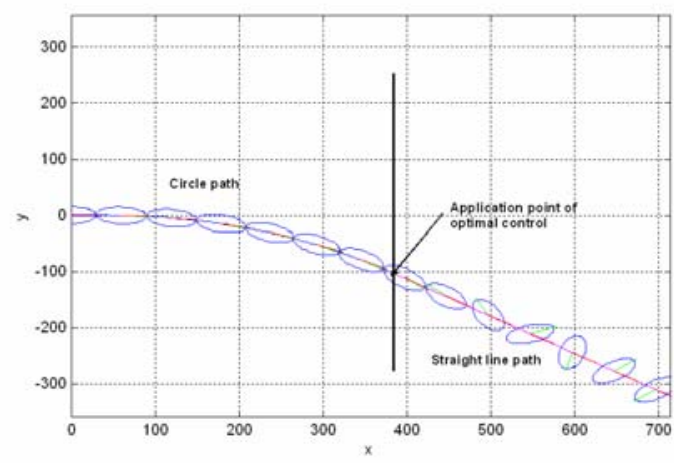

Figure10 airship configuration on circle line transition.

\section{Optimal transition of a non null curvature path} to a straight line

We now consider the issues pertaining to the switching between the non zero curvature path and a straight line. We have already characterized the forms of the optimal state and control trajectories in each mode separately. Therefore, we need to further specify the time interval corresponding to the mode switching and the parameters that determine when and for how long the singular control lasts. We proceed to address both issues using a continuity argument.We look for the fastest way for the airship to move from non null curvature paths to a straight line in optimal time. The line is characterized by zero values of $\beta$ and $r$, and the non null curvature paths are characterized by non zero values of $\beta$ and $r$. Let's use the dynamics of $\beta$ and $r$ for achieving this objective. In optimal control literature, the following theorem is demonstrated.

\section{Theorem:}

For any linear normal system the optimal control is of bang-bang type ${ }^{1}$.

The normality condition means that the system is controllable with respect to each of its control inputs. The determinant of the controllability matrix is:

$$
a_{21} b_{1}^{2}+b_{1} b_{2}\left(a_{22}-a_{11}\right)-a_{12} b_{2}^{2}
$$

The system under consideration is normal. Let's find the switching surface allowing the system to intersect the origin, starting from any initial condition within this surface, and under a specific control, i.e. $\delta_{r}= \pm 1$. The dynamics of the Yaw rate and the skid angle are asymptotically stable. To simplify the calculus, a separation of dynamics is performed by state matrix diagonalization, by means of linear state space variable transformation. Let

$$
\left(\begin{array}{l}
z_{1} \\
z_{2}
\end{array}\right)=T^{-1}\left(\begin{array}{l}
\beta \\
r
\end{array}\right)
$$

where $T$ is a $2 \times 2$ matrix formed by the eigenvectors corresponding to the system eigenvalues. The resultant diagonalized dynamics are given by:

$$
\begin{aligned}
& z_{1}=\lambda_{1} z_{1}+b_{z 1} \delta_{r} \\
& z_{2}=\lambda_{2} z_{2}+b_{z 2} \delta_{r}
\end{aligned}
$$

The corresponding solutions of this system are given by:

$$
\begin{aligned}
& z_{1}(t)=-b_{z 1} \frac{\delta_{r}}{\lambda_{1}}+\frac{e^{\lambda_{1} t}\left(b_{z 1} \delta_{r}+z_{10} \lambda_{1}\right)}{\lambda_{1}} \\
& z_{2}(t)=-b_{z 2} \frac{\delta_{r}}{\lambda_{2}}+\frac{e^{\lambda_{2} t}\left(b_{z 2} \delta_{r}+z_{20} \lambda_{2}\right)}{\lambda_{2}}
\end{aligned}
$$

where $z_{10}$ and $z_{20}$ are the initial conditions. To find the switching surface we look for the initial conditions from which the system dynamic trajectory cross the origin under the control $\delta_{r}= \pm 1$. The target point is $\left(\begin{array}{l}z_{1}\left(t_{c}\right) \\ z_{2}\left(t_{c}\right)\end{array}\right)=\left(\begin{array}{l}0 \\ 0\end{array}\right)$. However, the cross time with the origin $t_{c}$ is easily calculated from the system (51):

$$
t_{c}=\frac{\ln \left(\frac{b_{z 1} \delta_{r}}{b_{z 1} \delta_{r}+z_{10} \lambda_{1}}\right)}{\lambda_{1}}
$$

and

$$
t_{c}=\frac{\ln \left(\frac{b_{z 2} \delta_{r}}{b_{z 2} \delta_{r}+z_{20} \lambda_{2}}\right)}{\lambda_{2}}
$$

equalizing times and solving the resulting equation for $z_{10}$ we find:

$$
z_{10}=\frac{-\delta_{r}\left(\left(\frac{b_{z 2} \delta_{r}}{b_{z 2} \delta_{r}+z_{20} \lambda_{2}}\right)^{\frac{\lambda_{1}}{\lambda_{2}}}-1\right)}{\left(\frac{b_{z 2} \delta_{r}}{b_{z 2} \delta_{r}+z_{20} \lambda_{2}}\right)^{\frac{\lambda_{1}}{\lambda_{2}}}}
$$

this equation defines the switching surface in $z_{1}$ and $z_{2}$ coordinates corresponding to $\delta_{r}= \pm 1$, figure 11 and 12 , in figure 13 , we show the whole switching surface. 


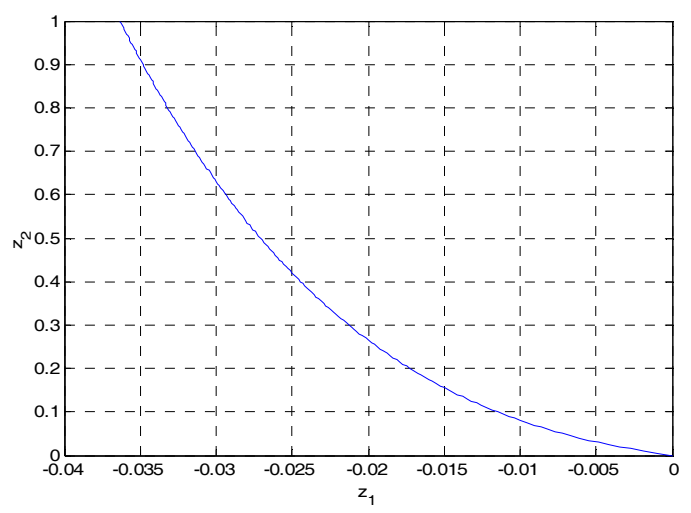

Figure11 switching surface for $\delta_{r}=1$

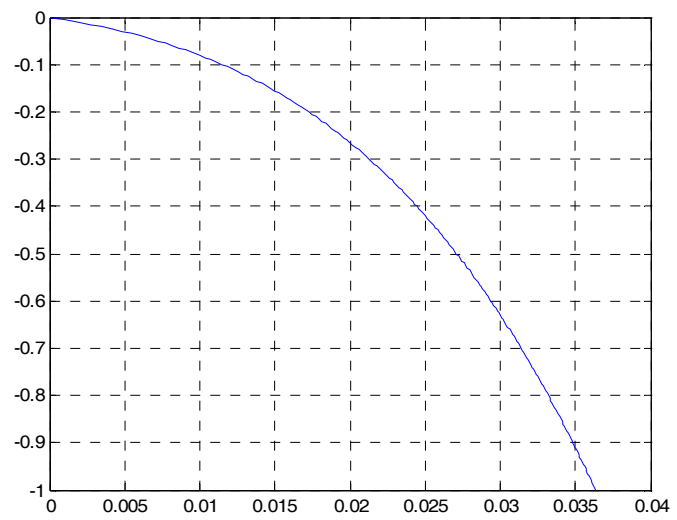

Figure12 Switching surface for $\delta_{r}=-1$

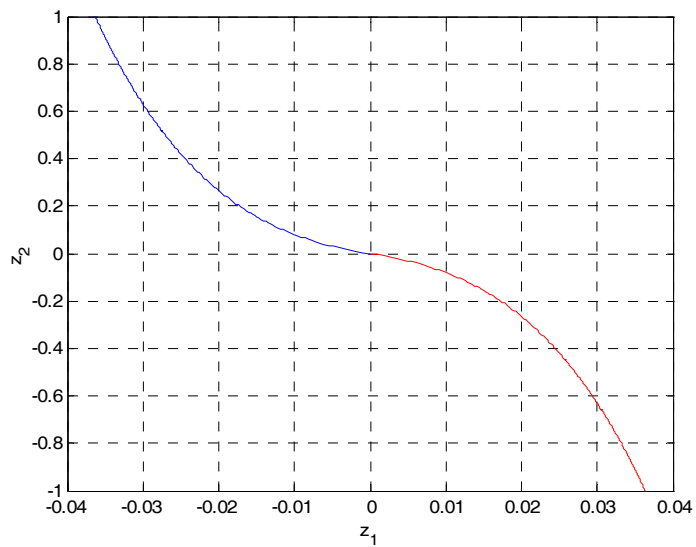

Figure13 Total switching surface

figure 14 shows the transition trajectories when applying a bang-bang control. The resultant trajectories have the hysteresis cycle form.

The algorithm of the optimal transition is based on the detection of the cross points of the bang-bang trajectories with the switching surface, the idea is to compare the horizontal distance of the system state point $\left(z_{1}, z_{2}\right)$ from the switching surface by replacing the $z_{2}$ in equation (54) and switching when the condition $\left|z_{1}-z_{10}\right|<\varepsilon$ is hold. In this case the control switch to the other bang. The same algorithm is applied to detect the intersection with the origin, when is the case, the control is hold to zero. Figure 15 show the resulting time-optimal transition control $\delta_{r}^{*}$ from a circle to a straight line. Figure 16 illustrate a comparison of $\beta$ response under application of $\delta_{r}^{*}$ and $\delta_{r}=0$. We see that the $\beta$ response to $\delta_{r}^{*}$ reaches the zero very fast.

In figure 17 we show the configuration of the airship on the resultant trajectory by application of $\delta_{r}^{*}$

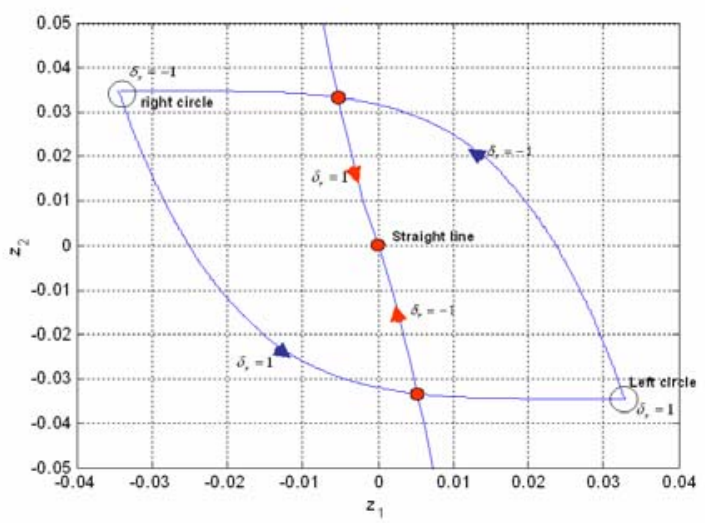

Figure14 State space optimal transition from circle to straight line

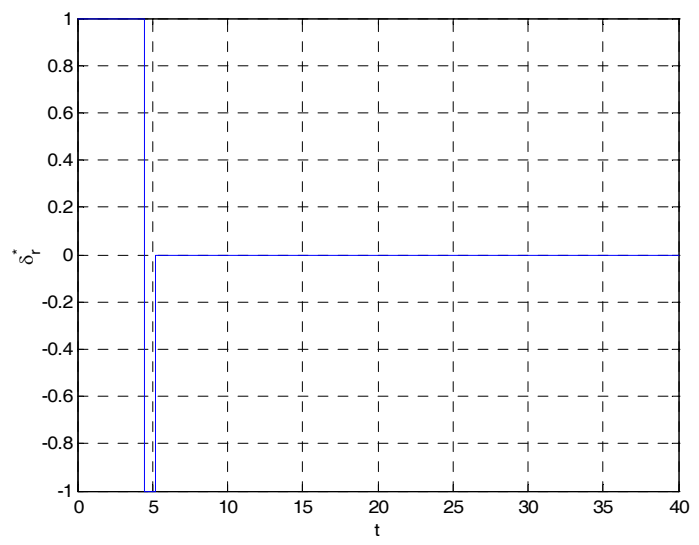

Figure15 Time-optimal circle to line transition control $\delta_{r}^{*}$ 


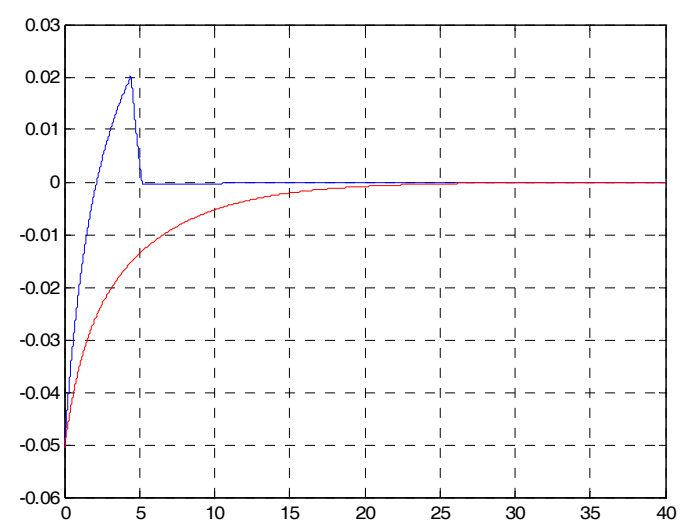

Figure16 skid angle $\beta$ response to $\delta_{r}^{*}$ and $\delta=0$

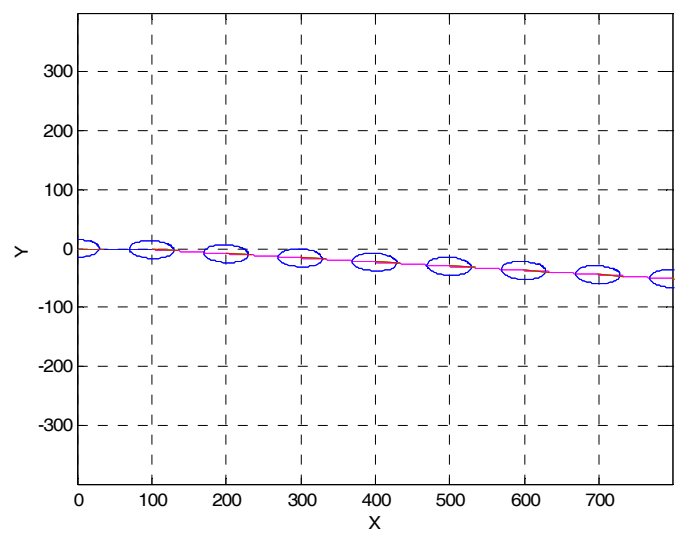

Figure17 Geometric configuration of airship response to $\delta_{r}^{*}$

\section{Conclusion and future work}

In this paper, a characterization of the time-optimal reference paths for lateral navigation of the airship is established, the Maximum Principle of Pontryagin gives a local information of the optimality of the paths. In the future work, this study should be completed by a geometric reasoning for providing a way to select inside of this family, the optimal path to link any two configurations in lateral plan.

\section{Acknowledgment}

The authors wish to thanks P. Souères and E. Hygounenc, from LAAS-CNRS (Toulouse, France).

\section{References}

${ }^{1}$ Athans, M., Falb, P. L., "Optimal Control, An introduction to the Theory and its Applications," McGRAW-HILL BOOK COMPANY, 1966.

${ }^{2}$ Bestaoui, Y., Hima, S., "Some Insights in Path Planning of Small Autonomous Airships," Journal of Archives of Control Sciences, Polish Academy of Sciences, vol. 11, 2001, pp; 139-166.
${ }^{3}$ Bryson, A.E., HO, Y.C., "Applied Optimal Control, Optimization, Estimation and Control," JOHN WILEY \& SONS, 1975.

${ }^{4}$ Fossen, T., "Guidance and Control of Ocean Vehicles," J. WILEY PRESS, 1996.

${ }^{5}$ Hygounenc, E., Souères, P., Lacroix, S., "Modélisation d'un Dirigeable Etude de la Cinématique et de la Dynamique, " LAAS-CNRS, 00426, Octobre 2000.

${ }^{6}$ Khoury, G.A., Gillet, J.D. Editors, "Airship Technology," CAMBRIDGE AEROSPACE, SERIE 10, 1999.

${ }^{7}$ Laumond, J.P, "Robot Motion Planning and Control," Lecture Notes in Control and Information Sciences 229, SPRINGER, 1998.

${ }^{8}$ Murray, R.M., Li, Z., Sastry, S., S., "Mathematical Introduction to Robotic Manipulation," CRC Press, 1998.

${ }^{9}$ Pontriagin, L., Boltianski, V., Gamkrélidzé, R., Michtchenko, E., "The Mathematical Theory of Optimal Processes," Interscience Publishers, 1962.

${ }^{10}$ Robbins, H. M., "A Generalized Legender-Clebsch Condition for the Singular Cases of Optimal Control," IBM Journal of Research and Development, Vol. 11, pp. 361-372, July 1967.

${ }^{11}$ Zefran, M., Kumer, V., Croke, C., "Metrics and Connections for Rigid-Body Kinematics," International Journal of Robotics Research, Vol. 18, \#2, pp. 243-258. feb. 1999. 